\title{
Enantioselective Double C-H Activation of Dihydronaphthalenes
}

\author{
Huw M. L. Davies* and Qihui Jin \\ Department of Chemistry, University at Buffalo, The State University of New York, \\ Buffalo, NY 14260
}

Supporting Information

Complete experimental data (S1-13) ad 1H-NMR spectra of 7 and 10a 


\section{General Procedure:}

${ }^{1} \mathrm{H}$ NMR spectra were run at either 400 or $500 \mathrm{MHz}$, and ${ }^{13} \mathrm{C}$ NMR at either 75 or 125 $\mathrm{MHz}$ with the sample solvent being $\mathrm{CDCl}_{3}$ unless otherwise noted. Mass spectral determinations were carried out in GC-MS (EI), LC-MS (ESI) or by Instrument Center, Department of Chemistry, University at Buffallo. IR spectra were obtained using a Perkin Elmer 1760X FT-IR. Optical rotations were measured using a Jasco DIP-370 digital polarimeter. Elemental analyses were performed by Atlantic Microlabs Inc., Norcross GA. Enantiomeric excess was determined by HPLC using a Chiralcel OD-H or $(S, S)$-Whelk-O 1 chiral analytical column (UV detection at $254 \mathrm{~nm}$ ). Analytical TLC was performed on $0.25 \mathrm{~mm}$ E. Merck silica gel (60F-254) plates using UV light.

Glassware was dried in oven overnight then flame or heat-gun dried prior to use. Reactions were conducted under argon atmosphere. Column chromatography was carried out on Merck silica gel 60 (230-400 mesh). Solvent THF, Et ${ }_{2} \mathrm{O}, \mathrm{CH}_{3} \mathrm{CN}, \mathrm{CH}_{2} \mathrm{Cl}_{2}$ and toluene were dried by solvent purifier. 2,2-Dimethylbutane (DMB) was purified by passing through silica gel 60 (Merck, 230-400 mesh, activated by heating under argon),

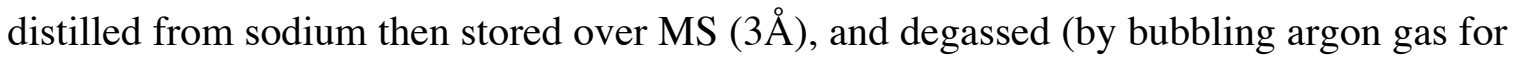
5-10 min) prior to use.

\section{Starting Materials:}

The following starting materials $5,{ }^{1} 6,{ }^{2} 9 \mathbf{a},{ }^{3} \mathbf{9 b},{ }^{4} \mathbf{1 1},{ }^{5} \mathbf{1 4},{ }^{6} \mathbf{1 7}^{5}$ and catalyst $\mathrm{Rh}_{2}(S \text {-DOSP })_{4}{ }^{7}$ were prepared according to the published procedures.

\footnotetext{
${ }^{1}$ Adamczyk, M.; Watt, D. A. and Netzel, D. A. J. Org. Chem. 1984, 49, 4226.

2 Davies, H. M. L.; Clark, T. J. and Smith, H. D. J. Org. Chem. 1991, 56, 3817.

${ }^{3}$ Harrowven, D. C. and Dainty, R. F. Tetrahedron, 1997, 53, 15771-15786.

${ }^{4}$ Magnus, P.; Lacour, J.; Coldham, I.; Mugrage, B. and Bauta, W. B. Tetrahedron 1995, 51, 11087.

${ }^{5}$ Davies, H. M. L. and Jin, Q. J. Am. Chem. Soc. 2004, 126, 10862.

${ }^{6}$ Davies, H. M. L. and Jin, Q. Prod. Natl. Acad. Sci. USA 2004, 101, 5472.

${ }^{7}$ Davies, H. M. L.; Bruzinski, P.; Hutcheson, D. K.; Kong, N. and Fall, M. J. J. Am. Chem. Soc. 1996, 118, 6897.
} 


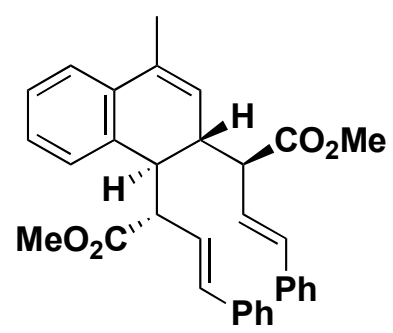

7: To a rapidly stirring solution of 1,2-dihydro-4-methylnaphthalene (5) (0.5 mmol) and $\mathrm{Rh}_{2}(S \text {-DOSP })_{4}(19 \mathrm{mg}, 0.01 \mathrm{mmol})$ in 2,2-dimethylbutane $(1 \mathrm{~mL})$ was added a solution of (E)-methyl phenylvinyldiazoacetate (6) $(202 \mathrm{mg}, 1.0 \mathrm{mmol})$ over a $2 \mathrm{~h}$ period via syringe-pump at RT. The mixture was stirred for an additional $5 \mathrm{~min}$. The solvent was evaporated under reduced pressure and the residue was purified by flash chromatography on silica gel (10:1 to $2: 1$ pentane/ether eluent) to provide the mono $\mathrm{C}-\mathrm{H}$ insertion product 8 (75 mg, 47\% yield) as a white solid and double C-H insertion product 7 (64 mg, 26\% yield) as a pale yellow solid.

7: pale yellow solid; $\mathrm{R}_{\mathrm{f}} 0.42(2: 1$ pentane/ether $) ;[\alpha]_{\mathrm{D}}^{25}+63.8^{\circ}\left(\right.$ c 2.70, $\left.\mathrm{CHCl}_{3}\right)$; FTIR $\left(\mathrm{CH}_{2} \mathrm{Cl}_{2}\right): 2919,2850,1733,1266,1162,739 \mathrm{~cm}^{-1} ;{ }^{1} \mathrm{H}$ NMR $\left(500 \mathrm{MHz}, \mathrm{CDCl}_{3}\right) \delta 7.37$ $(\mathrm{d}, J=7.3 \mathrm{~Hz}, 2 \mathrm{H}), 7.35-7.23(\mathrm{~m}, 10 \mathrm{H}), 7.17(\mathrm{td}, J=7.3,1.8 \mathrm{~Hz}, 1 \mathrm{H}), 7.04(\mathrm{~d}, J=7.3$ $\mathrm{Hz}, 1 \mathrm{H}), 6.39$ (d, $J=15.9 \mathrm{~Hz}, 1 \mathrm{H}), 6.30$ (d, $J=15.9 \mathrm{~Hz}, 1 \mathrm{H}), 6.19$ (dd, $J=15.9,9.8 \mathrm{~Hz}$, $1 \mathrm{H}), 6.00(\mathrm{dd}, J=15.9,9.8 \mathrm{~Hz}, 1 \mathrm{H}), 5.68$ (br d, $J=6.1 \mathrm{~Hz}, 1 \mathrm{H}), 3.61$ (s, 3H), 3.40 (s, 3H), 3.38 (appt. t, $J=9.8 \mathrm{~Hz}, 1 \mathrm{H}$ ), 3.22 (br d, $J=10.1 \mathrm{~Hz}, 1 \mathrm{H}$ ), 2.96 (appt. t, $J=9.8 \mathrm{~Hz}$, $1 \mathrm{H}), 2.87$ (br dd, $J=9.6,6.1 \mathrm{~Hz}, 1 \mathrm{H}), 2.08(\mathrm{~s}, 3 \mathrm{H}) ;{ }^{13} \mathrm{C} \mathrm{NMR}\left(125 \mathrm{MHz}, \mathrm{CDCl}_{3}\right) \delta$ $173.13,173.11,136.6,136.5,134.2$, 134.11, 134.10, 134.0, 133.8, 128.9, 128.52, 128.49, 127.72, 127.71, 127.5, 127.3, 126.4 (2C), 126.0, 125.4, 123.6, 123.2, 53.4, 52.1, 51.8, 51.4, 43.1, 37.5, 19.2; LC-MS (ESI) $m / z$ (relative intensity): 433.3 (12), $493.2\left([\mathrm{M}+\mathrm{H}]^{+}\right.$, 100), $515.1\left([\mathrm{M}+\mathrm{Na}]^{+}, 65\right)$; HRMS (ESI) $m / z$ Calcd for $\left[\mathrm{C}_{33} \mathrm{H}_{32} \mathrm{NaO}_{5}\right]\left([\mathrm{M}+\mathrm{Na}]^{+}\right)$: 515.2193. Found: 515.2204; HPLC analysis: 99\% ee (Chiralcel OD-H, 5.0\% $i$-PrOH in hexane, $0.8 \mathrm{~mL} / \mathrm{min}, \lambda=254 \mathrm{~nm}, \mathrm{t}_{\mathrm{R}}=8.8 \mathrm{~min}$, major; $\mathrm{t}_{\mathrm{R}}=13.4 \mathrm{~min}$, minor).

8: ${ }^{1} \mathrm{H}$ NMR $\left(500 \mathrm{MHz}, \mathrm{CDCl}_{3}\right) \delta$ 7.35-7.28 (m, 4H), 7.25-7.17 (m, 3H), $7.14(\mathrm{td}, J=7.0$, $1.8 \mathrm{~Hz}, 1 \mathrm{H}), 7.07$ (br d, $J=7.0 \mathrm{~Hz}, 1 \mathrm{H}), 6.41$ (d, $J=15.9 \mathrm{~Hz}, 1 \mathrm{H}), 6.14$ (dd, $J=15.9,9.8$ $\mathrm{Hz}, 1 \mathrm{H}), 5.73$ (br d, $J=3.0 \mathrm{~Hz}, 1 \mathrm{H}), 3.68$ (s, 3H), 3.10 (appt. t, $J=9.3 \mathrm{~Hz}, 1 \mathrm{H}), 2.87$ (dd, $J=14.2,6.0 \mathrm{~Hz}, 1 \mathrm{H}), 2.86-2.79(\mathrm{~m}, 1 \mathrm{H}), 2.71(\mathrm{dd}, J=14.2,6.5 \mathrm{~Hz}, 1 \mathrm{H}), 2.06(\mathrm{~s}, 3 \mathrm{H})$. 
The ${ }^{1} \mathrm{H}$ NMR is consistent with published data; ${ }^{5}$ HPLC analysis: 99\% ee (Chiralcel OD$\mathrm{H}, 2.0 \% i$-PrOH in hexane, $0.8 \mathrm{~mL} / \mathrm{min}, \lambda=254 \mathrm{~nm}, \mathrm{t}_{\mathrm{R}}=9.0 \mathrm{~min}$, major; $\mathrm{t}_{\mathrm{R}}=10.8 \mathrm{~min}$, minor).
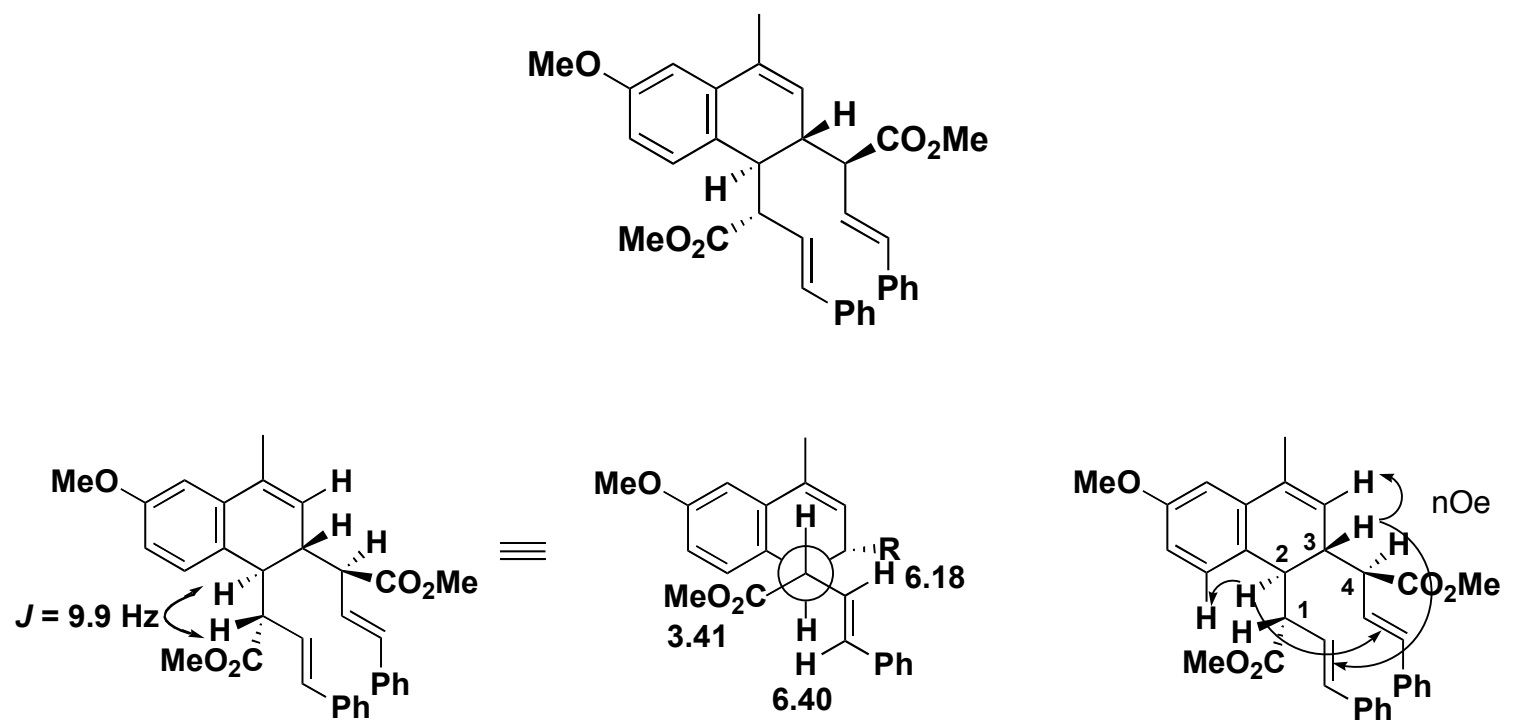

10a: To a refluxing solution of 1,2-dihydro-6-methoxy-4-methylnaphthalene (9a) (0.5 $\mathrm{mmol})$ and $\mathrm{Rh}_{2}(S \text {-DOSP })_{4}(19 \mathrm{mg}, 0.01 \mathrm{mmol})$ in 2,2-dimethylbutane $(1 \mathrm{~mL})$ was added a solution of (E)-methyl phenylvinyldiazoacetate (6) $(304 \mathrm{mg}, 1.5 \mathrm{mmol})$ over a $2 \mathrm{~h}$ period via syringe-pump. The mixture was refluxed for an additional $5 \mathrm{~min}$. The solvent was evaporated under reduced pressure and the residue was purified by flash chromatography on silica gel (2:1 pentane/ether eluent) to provide 10a (240 $\mathrm{mg}, 92 \%$ yield) as a pale yellow solid.

10a: pale yellow solid; $\mathrm{R}_{\mathrm{f}} 0.25$ (2:1 pentane/ether); $[\alpha]_{\mathrm{D}}^{25}+12.8^{\circ}\left(c 5.40, \mathrm{CHCl}_{3}\right)$; FTIR $\left(\mathrm{CH}_{2} \mathrm{Cl}_{2}\right): 3028,3059,3026,2949,2839,1733,1603,1572,1494,1449,1434,1320$, 1287, 1256, 1223, 1199, 1160, 1111, 1062, 1047, $1029 \mathrm{~cm}^{-1} ;{ }^{1} \mathrm{H}$ NMR (500 MHz, $\left.\mathrm{CDCl}_{3}\right)$ $\delta 7.37(\mathrm{~d}, J=7.3 \mathrm{~Hz}, 2 \mathrm{H}), 7.34-7.21(\mathrm{~m}, 8 \mathrm{H}), 6.97(\mathrm{~d}, J=8.2 \mathrm{~Hz}, 1 \mathrm{H}), 6.82(\mathrm{~d}, J=2.5$ $\mathrm{Hz}, 1 \mathrm{H}), 6.71(\mathrm{dd}, J=8.2,2.5 \mathrm{~Hz}, 1 \mathrm{H}), 6.40(\mathrm{~d}, J=15.8 \mathrm{~Hz}, 1 \mathrm{H}), 6.30$ (d, $J=15.8 \mathrm{~Hz}$, 1H), $6.18(\mathrm{dd}, J=15.8,9.8 \mathrm{~Hz}, 1 \mathrm{H}), 6.00(\mathrm{dd}, J=15.8,9.6 \mathrm{~Hz}, 1 \mathrm{H}), 5.70(\mathrm{br} \mathrm{d}, J=6.2$ $\mathrm{Hz}, 1 \mathrm{H}), 3.79$ (s, 3H), 3.60 (s, 3H), 3.41 (s, 3H), 3.36 (appt. t, $J=9.8 \mathrm{~Hz}, 1 \mathrm{H}$ ), 3.18 (br d, $J=9.9 \mathrm{~Hz}, 1 \mathrm{H}), 2.96$ (appt. t, $J=9.7 \mathrm{~Hz}, 1 \mathrm{H}), 2.86$ (br dd, $J=9.7,6.2 \mathrm{~Hz}, 1 \mathrm{H}), 2.06$ (s, 3H); ${ }^{13} \mathrm{C} \mathrm{NMR} \mathrm{(125} \mathrm{MHz,} \mathrm{CDCl}_{3}$ ) $\delta 173.12$ (C), 173.09 (C), 159.0 (C), 136.55 (C), 
$136.51(\mathrm{C}), 135.4(\mathrm{C}), 133.98(\mathrm{C}), 133.97(\mathrm{CH}), 133.66(\mathrm{CH}), 129.7(\mathrm{CH}), 128.5(\mathrm{CH})$, $128.4(\mathrm{CH}), 127.66(\mathrm{CH}), 127.65(\mathrm{CH}), 126.4(\mathrm{CH}, 2 \mathrm{C}), 126.1(\mathrm{C}), 126.0(\mathrm{CH}), 125.4$ $(\mathrm{CH}), 123.8(\mathrm{CH}), 111.7(\mathrm{CH}), 111.0(\mathrm{CH}), 55.1\left(\mathrm{CH}_{3}\right), 53.5(\mathrm{CH}), 52.0(\mathrm{CH}), 51.7$ $\left(\mathrm{CH}_{3}\right), 51.4\left(\mathrm{CH}_{3}\right), 42.3(\mathrm{CH}), 37.6(\mathrm{CH}), 19.2\left(\mathrm{CH}_{3}\right)$; LC-MS (ESI) $\mathrm{m} / z$ (relative intensity): $523.0\left([\mathrm{M}+\mathrm{H}]^{+}, 43\right), 545.0\left([\mathrm{M}+\mathrm{Na}]^{+}, 100\right)$; HRMS (ESI) $\mathrm{m} / \mathrm{z}$ Calcd for $\left[\mathrm{C}_{34} \mathrm{H}_{34} \mathrm{NaO}_{5}\right]^{+}$([M+Na $\left.]^{+}\right)$: 545.2298. Found: 545.2280; HPLC analysis: $99 \%$ ee (Chiralcel OD-H, $5.0 \% i$ PrOH in hexane, $0.8 \mathrm{~mL} / \mathrm{min}, \lambda=254 \mathrm{~nm}, \mathrm{t}_{\mathrm{R}}=9.7 \mathrm{~min}$, major; $\mathrm{t}_{\mathrm{R}}=15.0$ min, minor).

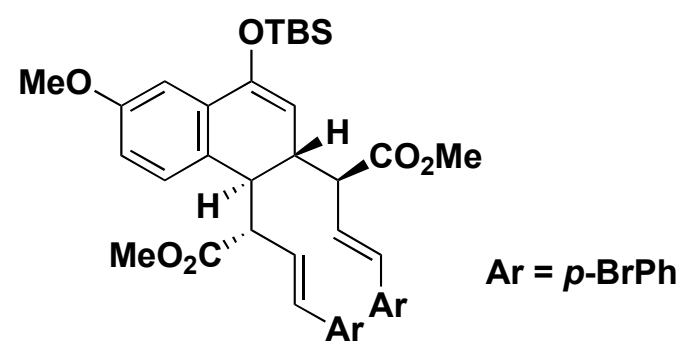

10b: To a refluxing solution of 4-tert-butyldimethylsilyloxy-1,2-dihydro-6-methoxynaphthalene (9b) $(0.5 \mathrm{mmol})$ and $\mathrm{Rh}_{2}(S \text {-DOSP })_{4}(19 \mathrm{mg}, 0.01 \mathrm{mmol})$ in 2,2dimethylbutane $(1 \mathrm{~mL})$ was added a solution of $(E)$-methyl pbromophenylvinyldiazoacetate ( $422 \mathrm{mg}, 1.5 \mathrm{mmol}$ ) over a $2 \mathrm{~h}$ period via syringe-pump. The mixture was refluxed for an additional $5 \mathrm{~min}$. The solvent was evaporated under reduced pressure and the residue was purified by flash chromatography on silica gel (2:1 pentane/ether eluent) to provide $10 \mathrm{~b}$ (343 $\mathrm{mg}, 86 \%$ yield) as a pale yellow solid.

10b: white solid (recrystallized from methanol), mp 93-96 ${ }^{\circ} \mathrm{C} ; \mathrm{R}_{\mathrm{f}} 0.43(2: 1$ pentane/ether); $[\alpha]_{\mathrm{D}}^{25}+17.0^{\circ}$ (c 4.00, $\left.\mathrm{CHCl}_{3}\right)$; FTIR $\left(\mathrm{CH}_{2} \mathrm{Cl}_{2}\right)$ : 2951, 2858, 1734, 1639, $1602,1575,1488,1464,1434,1362,1336,1261,1208,1160,1072,1038,1010 \mathrm{~cm}^{-1} ;{ }^{1} \mathrm{H}$ NMR (500 MHz, $\left.\mathrm{CDCl}_{3}\right) \delta 7.44(\mathrm{~d}, J=8.5 \mathrm{~Hz}, 2 \mathrm{H}), 7.42(\mathrm{~d}, J=8.5 \mathrm{~Hz}, 2 \mathrm{H}), 7.22(\mathrm{~d}, J=$ $8.5 \mathrm{~Hz}, 2 \mathrm{H}), 7.10(\mathrm{~d}, J=8.5 \mathrm{~Hz}, 2 \mathrm{H}), 7.06(\mathrm{~d}, J=2.4 \mathrm{~Hz}, 1 \mathrm{H}), 6.94(\mathrm{~d}, J=8.2 \mathrm{~Hz}, 1 \mathrm{H})$, $6.73(\mathrm{dd}, J=8.2,2.4 \mathrm{~Hz}, 1 \mathrm{H}), 6.32(\mathrm{~d}, J=15.8 \mathrm{~Hz}, 1 \mathrm{H}), 6.24(\mathrm{~d}, J=15.9 \mathrm{~Hz}, 1 \mathrm{H}), 6.17$ $(\mathrm{dd}, J=15.8,9.8 \mathrm{~Hz}, 1 \mathrm{H}), 5.97(\mathrm{dd}, J=15.9,9.6 \mathrm{~Hz}, 1 \mathrm{H}), 5.00(\mathrm{~d}, J=6.7 \mathrm{~Hz}, 1 \mathrm{H}), 3.79$ (s, 3H), 3.61 (s, 3H), 3.41 (s, 3H), 3.42 (appt. t, J = 9.8 Hz, 1H), 3.11 (br d, $J=9.8 \mathrm{~Hz}$, 1H), 2.98 (appt. t, $J=9.6 \mathrm{~Hz}, 1 \mathrm{H}), 2.85$ (br dd, $J=9.6,6.7 \mathrm{~Hz}, 1 \mathrm{H}), 1.03$ (s, 9H), 0.22 (s, 
3H), 0.20 (s, 3H); ${ }^{13} \mathrm{C}$ NMR (75 MHz, $\mathrm{CDCl}_{3}$ ) $\delta 173.0$ (C), 172.9 (C), 159.0 (C), 149.0 (C), $135.5(\mathrm{C}), 135.4(\mathrm{C}), 133.4(\mathrm{C}), 132.9(\mathrm{CH}), 132.6(\mathrm{CH}), 131.60(\mathrm{CH}), 131.56(\mathrm{CH})$, $129.5(\mathrm{CH}), 127.96(\mathrm{CH}), 127.90(\mathrm{CH}), 126.9(\mathrm{C}), 126.8(\mathrm{CH}), 126.2(\mathrm{CH}), 121.5(\mathrm{C}$, 2C), $113.6(\mathrm{CH}), 108.2(\mathrm{CH}), 103.0(\mathrm{CH}), 55.1\left(\mathrm{CH}_{3}\right), 53.6(\mathrm{CH}), 52.8(\mathrm{CH}), 51.8\left(\mathrm{CH}_{3}\right)$, $51.5\left(\mathrm{CH}_{3}\right), 41.9(\mathrm{CH}), 37.6(\mathrm{CH}), 25.8(\mathrm{C}), 18.2\left(\mathrm{CH}_{3}\right),-4.3\left(\mathrm{CH}_{3}\right),-4.9\left(\mathrm{CH}_{3}\right)$; HRMS (ESI) $\mathrm{m} / z$ Calcd for $\left[\mathrm{C}_{39} \mathrm{H}_{44} \mathrm{Br}_{2} \mathrm{NaO}_{6} \mathrm{Si}\right]^{+}\left([\mathrm{M}+\mathrm{Na}]^{+}\right): 817.1166$. Found: 817.1154; HPLC analysis: 99\% ee ((S,S)-Whelk-O 1, 15.0\% $i$-PrOH in hexane, $1.0 \mathrm{~mL} / \mathrm{min}, \lambda=254 \mathrm{~nm}, \mathrm{t}_{\mathrm{R}}$ $=13.4$ min, major; $\mathrm{t}_{\mathrm{R}}=16.4$ min, minor); Anal. Calcd for $\mathrm{C}_{39} \mathrm{H}_{44} \mathrm{Br}_{2} \mathrm{O}_{6} \mathrm{Si}: \mathrm{C}, 58.80 ; \mathrm{H}$, 5.57. Found: C, 58.88; H, 5.65.
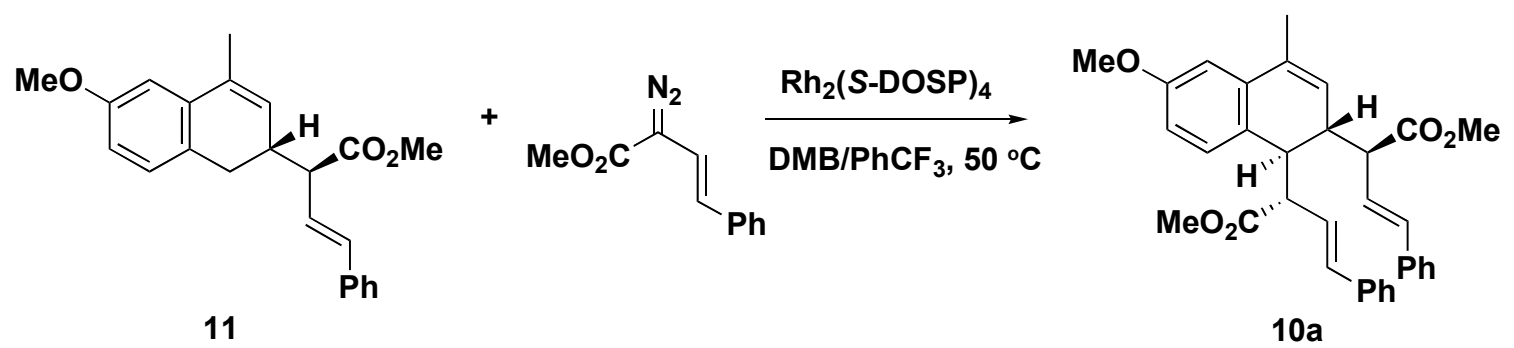

To a refluxing solution of $\mathbf{1 1}(35 \mathrm{mg}, 0.1 \mathrm{mmol})$ and $\mathrm{Rh}_{2}(S \text {-DOSP })_{4}(3.8 \mathrm{mg}, 0.002$ $\mathrm{mmol})$ in $\alpha, \alpha, \alpha$-trifluorotoluene $(0.2 \mathrm{~mL})$ and 2,2-dimethylbutane $(1.5 \mathrm{~mL})$ was added a solution of $(E)$-methyl phenylvinyldiazoacetate $(6)(41 \mathrm{mg}, 0.2 \mathrm{mmol})$ over a $2 \mathrm{~h}$ period via syringe-pump. The mixture was refluxed for an additional $5 \mathrm{~min}$. The solvent was evaporated under reduced pressure and the residue was purified by flash chromatography on silica gel (2:1 pentane/ether eluent) to provide 10a (47 $\mathrm{mg}, 90 \%$ yield) as a pale yellow solid.
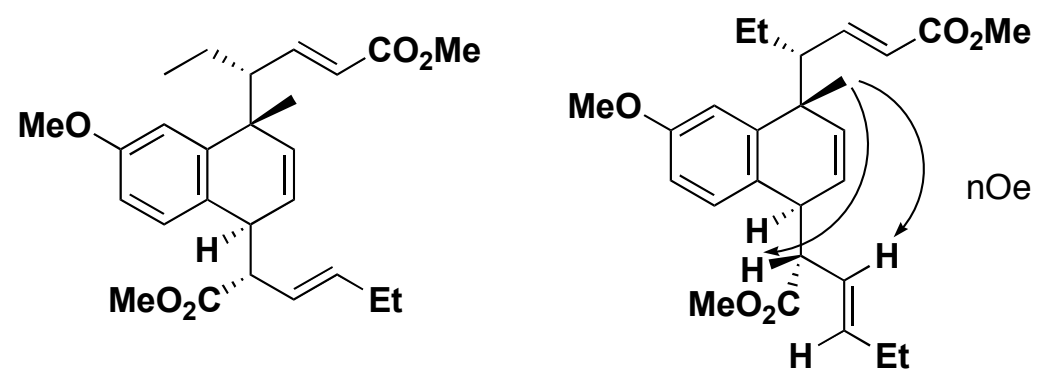
15: To a stirring solution of 1,2-dihydro-6-methoxy-4-methylnaphthalene (9a) (0.5 $\mathrm{mmol})$ and $\mathrm{Rh}_{2}(S \text {-DOSP })_{4}(19 \mathrm{mg}, 0.01 \mathrm{mmol})$ in DMB $(2 \mathrm{~mL})$ was added a solution of (E)-methyl 2-diazo-3-hexanoate $(308 \mathrm{mg}, 2 \mathrm{mmol})$ in DMB $(5 \mathrm{~mL})$ at RT over a $2 \mathrm{~h}$ period via syringe-pump. The resulting mixture was stirred for an additional $5 \mathrm{~min}$. The solvent was removed under reduced pressure and the residue was purified by flash chromatography on silica gel (5:1 pentane/ether eluent) to provide 15 (114 mg, 53\% yield).

15: colorless oil; $\mathrm{R}_{\mathrm{f}} 0.22$ (5:1 pentane/ether); $[\alpha]_{\mathrm{D}}{ }^{25}-81.3^{\circ}$ (c 2.60, $\mathrm{CHCl}_{3}$ ); FTIR (film): 2961, 2873, 1728, 1653, 1611, 1573, 1499, 1457, 1435, 1354, 1273, 1238, 1192, 1165 , 1047, $1016 \mathrm{~cm}^{-1} ;{ }^{1} \mathrm{H} \mathrm{NMR}\left(500 \mathrm{MHz}, \mathrm{CDCl}_{3}\right) \delta$ 7.15-7.11 (m, 1H), $6.88(\mathrm{dd}, J=15.6$, $10.4 \mathrm{~Hz}, 1 \mathrm{H}), 6.79-6.75(\mathrm{~m}, 2 \mathrm{H}), 5.96(\mathrm{~d}, J=15.6 \mathrm{~Hz}, 1 \mathrm{H}), 5.85(\mathrm{dd}, J=10.5,3.7 \mathrm{~Hz}$, $1 \mathrm{H}), 5.70(\mathrm{dd}, J=10.5,1.5 \mathrm{~Hz}, 1 \mathrm{H}), 5.30(\mathrm{br} \mathrm{dd}, J=15.3,9.5 \mathrm{~Hz}, 1 \mathrm{H}), 5.03$ (dt, $J=15.3$, $6.4 \mathrm{~Hz}, 1 \mathrm{H}), 3.95-3.91(\mathrm{~m}, 1 \mathrm{H}), 3.81$ (s, 3H), 3.77 (s, 3H), 3.74 (s, 3H), 3.31 (dd, $J=9.5$, $4.4 \mathrm{~Hz}, 1 \mathrm{H}), 2.40-2.31$ (m, 1H), 1.88-1.76 (m, 2H), 1.22 (s, 3H), 1.08-0.96 (m, 1H), 0.94$0.82(\mathrm{~m}, 1 \mathrm{H}), 0.75(\mathrm{t}, J=7.3 \mathrm{~Hz}, 3 \mathrm{H}), 0.58(\mathrm{t}, J=7.3 \mathrm{~Hz}, 3 \mathrm{H}) ;{ }^{13} \mathrm{C}$ NMR $(75 \mathrm{MHz}$, $\left.\mathrm{CDCl}_{3}\right) \delta 173.9,166.6,158.1,149.6,143.6,136.7,131.3,128.6,128.2,124.7,123.7$, $122.5,111.6,110.5,56.5,55.9,55.2,51.8,51.4,41.9,40.7,29.9,25.4,22.7,13.1,12.5$; LRMS (ESI) $m / z$ (relative intensity): 227.0 (22), 449.1 ([M+Na $\left.]^{+}, 100\right)$; HRMS (ESI) $m / z$ Calcd for $\left[\mathrm{C}_{26} \mathrm{H}_{34} \mathrm{NaO}_{5}\right]^{+}\left([\mathrm{M}+\mathrm{Na}]^{+}\right)$: 449.2298. Found: 449.2298; Enantiomeric excess was determined by converting to compound $\mathbf{1 6}$.

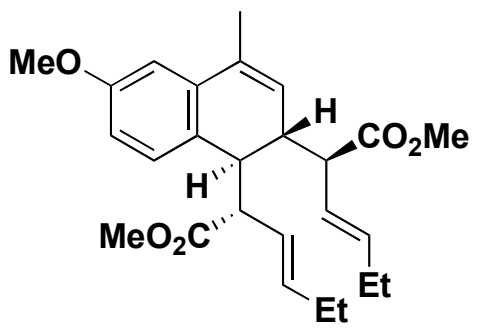

16: A solution of $\mathbf{1 5}(61 \mathrm{mg})$ in toluene $(3 \mathrm{~mL})$ was refluxed for $6 \mathrm{~h}$. The solvent was removed under reduced pressure and the residue was purified by flash chromatography 
on silica gel (4:1 to $2: 1$ pentane/ether) to give the product 16 (42 $\mathrm{mg}, 68 \%$ yield) as a colorless oil:

16: colorless oil; $\mathrm{R}_{\mathrm{f}} 0.18$ (5:1 pentane/ether); $[\alpha]_{\mathrm{D}}{ }^{25}+136.8^{\circ}$ (c 1.50, $\left.\mathrm{CHCl}_{3}\right)$; FTIR (film): 2962, 1735, 1604, 1572, 1492, 1434, 1255, 1200, 1158, $1049 \mathrm{~cm}^{-1} ;{ }^{1} \mathrm{H}$ NMR (500 MHz, $\left.\mathrm{CDCl}_{3}\right) \delta 6.88(\mathrm{~d}, J=8.2 \mathrm{~Hz}, 1 \mathrm{H}), 6.81(\mathrm{~d}, J=2.6 \mathrm{~Hz}, 1 \mathrm{H}), 6.65(\mathrm{dd}, J=8.2,2.6 \mathrm{~Hz}$, $1 \mathrm{H}), 5.60$ (br d, $J=5.0 \mathrm{~Hz}, 1 \mathrm{H}), 5.55(\mathrm{dt}, J=15.3,6.3 \mathrm{~Hz}, 1 \mathrm{H}), 5.43(\mathrm{dt}, J=15.3,6.3$ $\mathrm{Hz}, 1 \mathrm{H}$ ), 5.38 (br dd, $J=15.3,9.5 \mathrm{~Hz}, 1 \mathrm{H}), 5.23$ (br dd, $J=15.3,9.0 \mathrm{~Hz}, 1 \mathrm{H}), 3.80$ (s, 3H), 3.61 (s, 3H), 3.40 (s, 3H), 3.11 (appt. t, $J=9.9 \mathrm{~Hz}, 1 \mathrm{H}), 3.00$ (d, $J=10.4 \mathrm{~Hz}, 1 \mathrm{H})$, 2.73-2.64 (m, 2H), 2.11-1.99 (m, 4H), $2.04(\mathrm{~s}, 3 \mathrm{H}), 1.00(\mathrm{t}, J=7.3 \mathrm{~Hz}, 3 \mathrm{H}), 0.98(\mathrm{t}, J=$ $7.3 \mathrm{~Hz}, 3 \mathrm{H}) ;{ }^{13} \mathrm{C} \mathrm{NMR}\left(75 \mathrm{MHz}, \mathrm{CDCl}_{3}\right) \delta 174.1,174.0,158.8,137.2,137.0,135.3$, $133.4,129.7,126.6,125.5,124.9,124.4,111.4,109.9,55.2,52.9,51.54,51.47,51.2$, 41.7, 37.1, 15.5 (2C), 19.2, 13.52, 13.46; LRMS (ESI) $\mathrm{m} / z$ (relative intensity): 298.3 (29), 341.3 (22), 384.4 (13), $427.4\left([\mathrm{M}+\mathrm{H}]^{+}, 5\right), 449.3$ ([M+Na $\left.]^{+}, 100\right)$; HRMS (ESI) $m / z$ Calcd for $\left[\mathrm{C}_{26} \mathrm{H}_{34} \mathrm{NaO}_{5}\right]^{+}\left([\mathrm{M}+\mathrm{Na}]^{+}\right)$: 449.2298. Found: 449.2299; HPLC analysis: $99.4 \%$ ee (Chiralapk AD-RH, 0.5\% $i$-PrOH in hexane, $1.0 \mathrm{~mL} / \mathrm{min}, \lambda=254 \mathrm{~nm}, \mathrm{t}_{\mathrm{R}}=8.3 \mathrm{~min}$, minor; $\mathrm{t}_{\mathrm{R}}=9.8 \mathrm{~min}$, major); Anal. Calcd for $\mathrm{C}_{26} \mathrm{H}_{34} \mathrm{O}_{5}: \mathrm{C}, 73.21 ; \mathrm{H}, 8.03$. Found: $\mathrm{C}$, $73.00 ; \mathrm{H}, 8.07$.

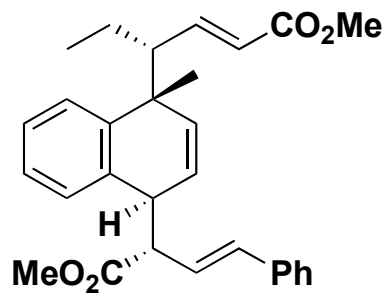

18: To a solution of stirring of $(R, 2 E)$-Methyl 4-( $(S)$-1,4-dihydro-1-methylnaphthalen-1yl)hex-2-enoate (17) $(135 \mathrm{mg}, 0.5 \mathrm{mmol})$ and $\mathrm{Rh}_{2}(S \text {-DOSP })_{4}(19 \mathrm{mg}, 0.01 \mathrm{mmol})$ in DMB (2 mL) was added a solution of (E)-methyl phenyldiazoacetate (6) $(202 \mathrm{mg}, 1$ $\mathrm{mmol})$ in $\mathrm{DMB}(5 \mathrm{~mL})$ at $\mathrm{RT}$ over a $2 \mathrm{~h}$ period via syringe-pump. The resulting mixture was stirred for an additional $5 \mathrm{~min}$. The solvent was removed under reduced pressure and the residue was purified by flash chromatography on silica gel (4:1-2:1 pentane/ether eluent) to provide $\mathbf{1 8}$ (138 $\mathrm{mg}, 62 \%$ yield). 
18: colorless oil; $\mathrm{R}_{\mathrm{f}} 0.38$ (2:1 pentane/ether); FTIR (film): 3027, 2960, 2873, 1727, 1654, 1490, 1436, 1355, 1270, 1242, 1194, 1164, $1140 \mathrm{~cm}^{-1} ;{ }^{1} \mathrm{H}$ NMR (500 MHz, $\left.\mathrm{CDCl}_{3}\right) \delta$ 7.30-7.26 (m, 1H), 7.24-7.15 (m, 5H), $7.15-7.10$ (m, 3H), 6.88 (dd, J = 15.6, 10.2 Hz, $1 \mathrm{H}), 6.12(\mathrm{dd}, J=15.9,9.5 \mathrm{~Hz}, 1 \mathrm{H}), 5.94(\mathrm{dd}, J=10.7,3.7 \mathrm{~Hz}, 1 \mathrm{H}), 5.93$ (d, $J=15.6 \mathrm{~Hz}$, $1 \mathrm{H}), 5.84(\mathrm{~d}, J=15.9 \mathrm{~Hz}, 1 \mathrm{H}), 5.77(\mathrm{dd}, J=10.7,1.5 \mathrm{~Hz}, 1 \mathrm{H}), 4.13-4.09$ (m, 1H), 3.77 (s, $3 \mathrm{H}), 3.75$ (s, 3H), 3.57 (dd, $J=9.5,4.3 \mathrm{~Hz}, 1 \mathrm{H}), 2.34$ (appt. td, $J=10.7,2.1 \mathrm{~Hz}, 1 \mathrm{H}$ ), $1.12(\mathrm{~s}, 3 \mathrm{H}), 1.07-1.00(\mathrm{~m}, 1 \mathrm{H}), 0.90-0.81(\mathrm{~m}, 1 \mathrm{H}), 0.56(\mathrm{t}, J=7.3 \mathrm{~Hz}, 3 \mathrm{H}) ;{ }^{13} \mathrm{C} \mathrm{NMR}$ (75 MHz, CDCl3) $\delta$ 173.4, 166.6, 149.6, 142.3, 136.6, 135.4, 133.6, 132.1, 128.3, 127.6, 127.3, 126.9, 126.2, 126.0, 125.2, 124.2, 123.8, 123.7, 56.5, 56.2, 52.0, 51.4, 41.7, 41.6, 29.8, 22.8, 12.5; LRMS (ESI) $m / z$ (relative intensity): $445.1\left([\mathrm{M}+\mathrm{H}]^{+}, 8\right), 467.1$ $\left([\mathrm{M}+\mathrm{Na}]^{+}, 100\right)$; HRMS (ESI) $m / z$ Calcd for $\left[\mathrm{C}_{29} \mathrm{H}_{32} \mathrm{NaO}_{4}\right]^{+}\left([\mathrm{M}+\mathrm{Na}]^{+}\right): 467.2193$. Found: 467.2203 .

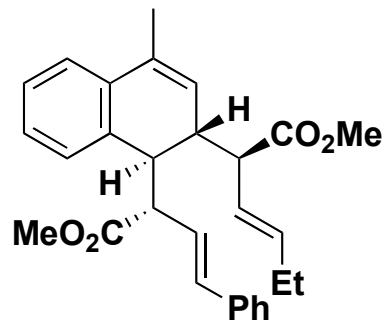

19: A solution of $\mathbf{1 8}(71 \mathrm{mg})$ in toluene $(4 \mathrm{~mL})$ was refluxed for $6 \mathrm{~h}$. The toluene was removed under reduced pressure and the residue was purified by flash chromatography on silica gel (5:1 pentane/ether eluent) to provide the product (61 $\mathrm{mg}, 86 \%$ yield) as a white solid.

19: white solid, $\mathrm{mp} 110-111{ }^{\circ} \mathrm{C} ; \mathrm{R}_{\mathrm{f}} 0.46$ (2:1 pentane/ether); $[\alpha]_{\mathrm{D}}{ }^{25}+111.5^{\circ}(c 4.50$, $\left.\mathrm{CHCl}_{3}\right)$; FTIR $\left(\mathrm{CH}_{2} \mathrm{Cl}_{2}\right): 3059,3028,2952,1734,1489,1450,1434,1330,1269,1228$, 1193, 1160, $1028 \mathrm{~cm}^{-1} ;{ }^{1} \mathrm{H}$ NMR (500 MHz, $\mathrm{CDCl}_{3}$ ) $\delta$ 7.40-7.35 (m, 2H), 7.35-7.30 (m, 2H), 7.28-7.21 (m, 3H), $7.14(\mathrm{td}, J=7.3,1.5 \mathrm{~Hz}, 1 \mathrm{H}), 7.00(\mathrm{~d}, J=7.3 \mathrm{~Hz}, 1 \mathrm{H}), 6.38(\mathrm{~d}, J$ $=15.9 \mathrm{~Hz}, 1 \mathrm{H}), 6.18(\mathrm{dd}, J=15.9,9.5 \mathrm{~Hz}, 1 \mathrm{H}), 5.63(\mathrm{br} \mathrm{d}, J=5.5 \mathrm{~Hz}, 1 \mathrm{H}), 5.44$ (dt, $J=$ 15.3, 6.4 Hz, 1H), 5.23 (br dd, $J=15.3,9.8 \mathrm{~Hz}, 1 \mathrm{H}), 3.58$ (s, 3H), 3.42 (s, 3H), 3.36 (appt. t, $J=9.9 \mathrm{~Hz}, 1 \mathrm{H}), 3.21(\mathrm{~d}, J=10.1 \mathrm{~Hz}, 1 \mathrm{H}), 2.76-2.67(\mathrm{~m}, 2 \mathrm{H}), 2.06(\mathrm{~s}, 3 \mathrm{H}), 2.06-$ $1.99(\mathrm{~m}, 2 \mathrm{H}), 0.98(\mathrm{t}, J=7.3 \mathrm{~Hz}, 3 \mathrm{H}) ;{ }^{13} \mathrm{C} \mathrm{NMR}\left(75 \mathrm{MHz}, \mathrm{CDCl}_{3}\right) \delta 173.7$ (C), 173.3 
(C), $137.4(\mathrm{CH}), 136.6(\mathrm{C}), 134.2(\mathrm{C}), 134.1(\mathrm{C}), 133.7(\mathrm{C}), 133.6(\mathrm{CH}), 128.8(\mathrm{CH})$, $128.5(\mathrm{CH}), 127.6(\mathrm{CH}), 127.4(\mathrm{CH}), 127.2(\mathrm{CH}), 126.4(\mathrm{CH}), 126.1(\mathrm{CH}), 124.6(\mathrm{CH})$, $123.6(\mathrm{CH}), 123.4(\mathrm{CH}), 53.5(\mathrm{CH}), 51.60(\mathrm{CH}), 51.56\left(\mathrm{CH}_{3}\right), 51.4\left(\mathrm{CH}_{3}\right), 42.8(\mathrm{CH})$, $37.2(\mathrm{CH}), 25.5\left(\mathrm{CH}_{2}\right), 19.2\left(\mathrm{CH}_{3}\right), 13.5\left(\mathrm{CH}_{3}\right)$; LRMS (ESI) $\mathrm{m} / z$ (relative intensity): $445.1\left([\mathrm{M}+\mathrm{H}]^{+}, 25\right), 467.1\left([\mathrm{M}+\mathrm{Na}]^{+}, 100\right)$; HRMS (ESI) $\mathrm{m} / z$ Calcd for $\left[\mathrm{C}_{29} \mathrm{H}_{32} \mathrm{NaO}_{4}\right]^{+}$ $\left([\mathrm{M}+\mathrm{Na}]^{+}\right)$: 467.2193. Found: 467.2208; Anal. Calcd for $\mathrm{C}_{29} \mathrm{H}_{32} \mathrm{O}_{4}: \mathrm{C}, 78.35 ; \mathrm{H}, 7.26$.

Found: C, 78.30; H, 7.26.

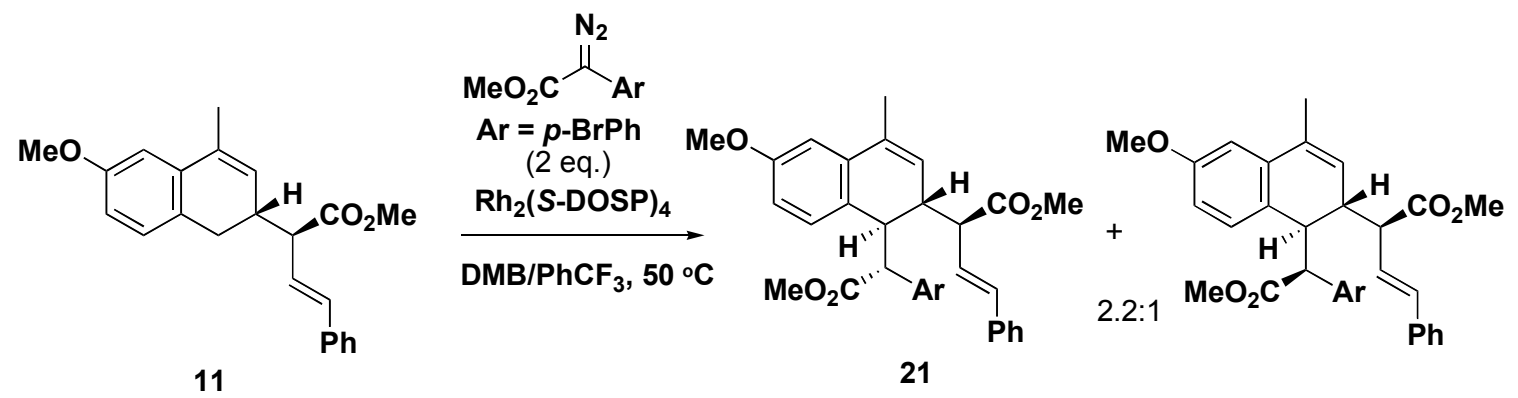

To a refluxing solution of $\mathbf{1 1}(70 \mathrm{mg}, 0.2 \mathrm{mmol})$ and $\mathrm{Rh}_{2}(S \text {-DOSP })_{4}$ (7.6 mg, 0.004 $\mathrm{mmol})$ in $\alpha, \alpha, \alpha$-trifluorotoluene $(0.2 \mathrm{~mL})$ and 2,2-dimethylbutane $(1.5 \mathrm{~mL})$ was added a solution of methyl $p$-bromophenyldiazoacetate $(102 \mathrm{mg}, 0.4 \mathrm{mmol})$ over a $2 \mathrm{~h}$ period via syringe-pump. The mixture was refluxed for an additional $5 \mathrm{~min}$. The solvent was evaporated under reduced pressure and the residue was purified by flash chromatography on silica gel (2:1 pentane/ether eluent) to provide $\mathbf{2 1}(99 \mathrm{mg}, 86 \%$ yield) as a mixture of two diastereomers in 2.2:1 ratio.

21 (mixture of two diastereomers): $\mathrm{R}_{\mathrm{f}} 0.32$ (2:1 pentane/ether); FTIR $\left(\mathrm{CH}_{2} \mathrm{Cl}_{2}\right): 3026$, 2951, 1839, 1733, 1604, 1573, 1490, 1435, 1333, 1257, 1198, 1160, $1011 \mathrm{~cm}^{-1}$; major: ${ }^{1} \mathrm{H}$ NMR (500 MHz, $\left.\mathrm{CDCl}_{3}\right)$ d $7.46(\mathrm{~d}, J=8.4 \mathrm{~Hz}, 2 \mathrm{H}), 7.37-7.31(\mathrm{~m}, 2 \mathrm{H}), 7.29-7.25(\mathrm{~m}$, $3 \mathrm{H}), 7.23(\mathrm{~d}, J=8.4 \mathrm{~Hz}, 2 \mathrm{H}), 7.07(\mathrm{~d}, J=8.5 \mathrm{~Hz}, 1 \mathrm{H}), 6.85(\mathrm{~d}, J=2.4 \mathrm{~Hz}, 1 \mathrm{H}), 6.72(\mathrm{dd}$, $J=8.5,2.4 \mathrm{~Hz}, 1 \mathrm{H}), 6.25(\mathrm{~d}, J=15.9 \mathrm{~Hz}, 1 \mathrm{H}), 5.80(\mathrm{dd}, J=15.9,9.9 \mathrm{~Hz}, 1 \mathrm{H}), 5.57(\mathrm{br}$ d, $J=6.4 \mathrm{~Hz}, 1 \mathrm{H}), 3.99$ (s, 3H), 3.68 (d, $J=11.3 \mathrm{~Hz}, 1 \mathrm{H}), 3.56$ (s, 3H), 3.43 (br d, $J=$ $11.3 \mathrm{~Hz}, 1 \mathrm{H}$ ), 3.35 (s, 3H), 2.83 (appt. t, $J=9.9 \mathrm{~Hz}, 1 \mathrm{H}$ ), 2.26 (br dd, $J=10.1,6.4 \mathrm{~Hz}$, $1 \mathrm{H}), 2.10(\mathrm{~s}, 3 \mathrm{H}) ;{ }^{13} \mathrm{C} \mathrm{NMR}\left(75 \mathrm{MHz}, \mathrm{CDCl}_{3}\right) \delta 173.1,173.0,159.2,136.5,136.2,134.0$ (2C), 131.8, 130.3, 130.2, 128.6, 127.8, 126.4, 126.2, 125.3, 123.5, 121.7, 111.8, 110.3, 
55.2, 54.3, 51.72, 51.67, 51.4, 42.7, 37.5, 19.2; minor: ${ }^{1} \mathrm{H}$ NMR (500 MHz, $\left.\mathrm{CDCl}_{3}\right) \delta$ 7.37-7.31 (m, 3H), 7.29-7.25 (m, 2H), $7.20(\mathrm{~d}, J=8.4 \mathrm{~Hz}, 2 \mathrm{H}), 6.78(\mathrm{~d}, J=2.7 \mathrm{~Hz}, 1 \mathrm{H})$, $6.75(\mathrm{~d}, J=8.4 \mathrm{~Hz}, 2 \mathrm{H}), 6.32(\mathrm{dd}, J=8.5,2.7 \mathrm{~Hz}, 1 \mathrm{H}), 6.30(\mathrm{~d}, J=15.9 \mathrm{~Hz}, 1 \mathrm{H}), 6.12$ (dd, $J=15.9,9.8 \mathrm{~Hz}, 1 \mathrm{H}), 6.04(\mathrm{~d}, J=8.5 \mathrm{~Hz}, 1 \mathrm{H}), 5.79-5.75(\mathrm{~m}, 1 \mathrm{H}), 3.73(\mathrm{~s}, 3 \mathrm{H}), 3.72$ (s, 3H), 3.70 (d, $J=11.3 \mathrm{~Hz}, 1 \mathrm{H}), 3.65$ (s, 3H), 3.31 (br d, $J=11.3 \mathrm{~Hz}, 1 \mathrm{H}), 2.94$ (appt. t, $J=9.9 \mathrm{~Hz}, 1 \mathrm{H}), 2.73$ (br dd, $J=10.1,6.4 \mathrm{~Hz}, 1 \mathrm{H}), 2.10$ (s, 3H); ${ }^{13} \mathrm{C}$ NMR $(75 \mathrm{MHz}$, $\left.\mathrm{CDCl}_{3}\right) \delta 173.3,173.2,158.8,136.8,136.6,135.0,133.9,133.4,131.1,130.9,128.8$, 128.5, 127.7, 126.5, 125.8, 124.8, 124.0, 121.1, 111.1, 109.9, 55.1, 53.4, 52.3, 52.1, 51.8, 43.8, 39.6, 19.2; LRMS (ESI) $\mathrm{m} / z$ (relative intensity): 130.1 (43), 281.0 (47), 351.0 (31), $575.2([\mathrm{M}+\mathrm{H}]+, 8), 597.1\left([\mathrm{M}+\mathrm{Na}]^{+}, 98\right), 599.1$ (100); HRMS (ESI) $\mathrm{m} / z$ Calcd for $\left[\mathrm{C}_{32} \mathrm{H}_{31}{ }^{81} \mathrm{BrNaO}_{4}\right]^{+}\left([\mathrm{M}+\mathrm{Na}]^{+}\right): 599.1227$. Found: 599.1217; Anal. Calcd for $\mathrm{C}_{32} \mathrm{H}_{31} \mathrm{BrO}_{5}$ : C, 66.79; H, 5.43. Found: C, 66.49; H, 5.43.

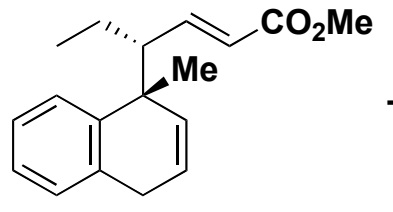

17

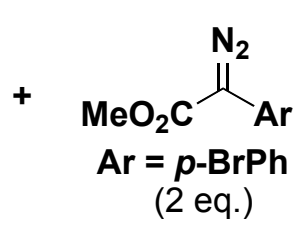

(2 eq.)

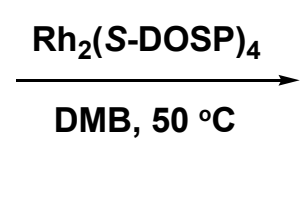

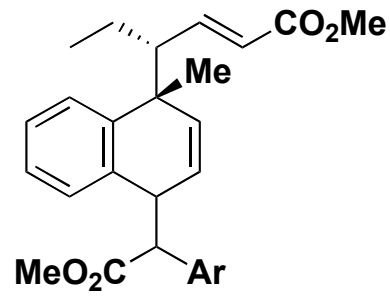

22 20:5:2:1 d.r.

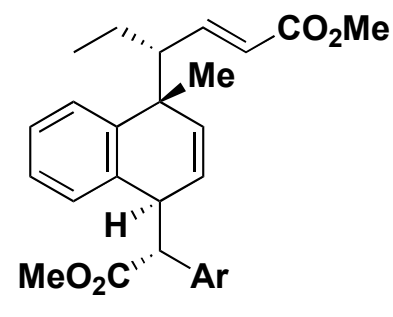

A

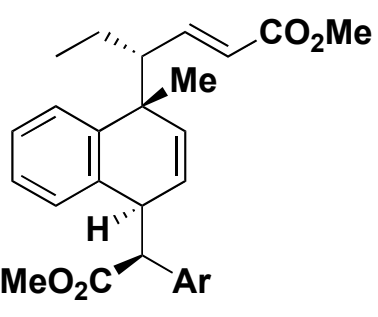

B
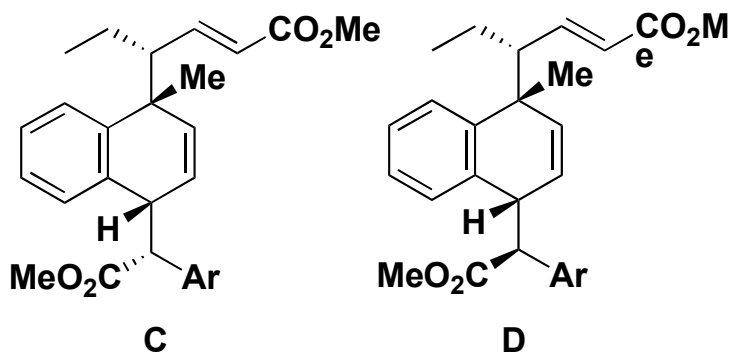

$\mathbf{A}: \mathbf{B}: \mathbf{C}: \mathbf{D}=20: 5: 2: 1$

To a solution of $17(0.5 \mathrm{mmol})$ and $\mathrm{Rh}_{2}(S-\mathrm{DOSP})_{4}(19 \mathrm{mg}, 0.01 \mathrm{mmol})$ in 2,2dimethylbutane $(1 \mathrm{~mL})$ was added a solution of methyl $p$-bromophenyldiazoacetate (255 $\mathrm{mg}, 1 \mathrm{mmol}$ ) over a $2 \mathrm{~h}$ period via syringe-pump. The mixture was stirred for an 
additional $5 \mathrm{~min}$. The solvent was evaporated under reduced pressure and the residue was purified by flash chromatography on silica gel (4:1 pentane/ether eluent) to provide 22 (200 mg, 80\% yield) as a mixture of four diastereomers in a 20:5:2:1 ratio.

22 (mixture of four diastereomers): $\mathrm{R}_{\mathrm{f}} 0.40$ (2:1 pentane/ether); FTIR $\left(\mathrm{CH}_{2} \mathrm{Cl}_{2}\right): 3028$, 2958, 2873, 1724, 1653, 1489, 1435, 1408, 1357, 1271, 1241, 1194, 1171, 1140, 1074, 1011 ;

A: ${ }^{1} \mathrm{H}$ NMR $\left(500 \mathrm{MHz}, \mathrm{CDCl}_{3}\right)$ ठ 7.34-7.31 (m, 1H), 7.24-7.19 (m, 4H), 7.17 -7.13 (m, $1 \mathrm{H}), 6.82(\mathrm{dd}, J=15.6,10.3 \mathrm{~Hz}, 1 \mathrm{H}), 6.73(\mathrm{~d}, J=8.6 \mathrm{~Hz}, 2 \mathrm{H}), 5.98(\mathrm{dd}, J=10.7,4.0 \mathrm{~Hz}$, $1 \mathrm{H}), 5.90(\mathrm{~d}, J=15.6 \mathrm{~Hz}, 1 \mathrm{H}), 5.63(\mathrm{dd}, J=10.7,1.0 \mathrm{~Hz}, 1 \mathrm{H}), 4.26$ (appt. t, $J=4.0 \mathrm{~Hz}$, $1 \mathrm{H}), 3.96(\mathrm{~d}, J=5.2 \mathrm{~Hz}, 1 \mathrm{H}), 3.74(\mathrm{~s}, 3 \mathrm{H}), 3.71(\mathrm{~s}, 3 \mathrm{H}), 2.28(\mathrm{td}, J=10.3,2.1 \mathrm{~Hz}, 1 \mathrm{H})$, 1.02-0.93 (m, 1H), 0.82-0.72 (m, 1H), $0.60(\mathrm{~s}, 3 \mathrm{H}), 0.53(\mathrm{t}, J=7.3 \mathrm{~Hz}, 3 \mathrm{H}) ;{ }^{13} \mathrm{C}$ NMR (75 MHz, $\mathrm{CDCl}_{3}$ ) $\delta 173.0$ (C), $166.5(\mathrm{C}), 149.4(\mathrm{CH}), 142.3$ (C), 135.3 (C), 134.1 (C), $132.3(\mathrm{CH}), 131.3(\mathrm{CH}), 130.5(\mathrm{CH}), 127.7(\mathrm{CH}), 127.0(\mathrm{CH}), 125.9(\mathrm{CH}), 125.2(\mathrm{CH})$, $124.4(\mathrm{CH}), 123.8(\mathrm{CH}), 121.2(\mathrm{C}), 58.5(\mathrm{CH}), 56.1(\mathrm{CH}), 52.0\left(\mathrm{CH}_{3}\right), 51.4\left(\mathrm{CH}_{3}\right), 41.6$ $(\mathrm{CH}), 41.5(\mathrm{C}), 29.2\left(\mathrm{CH}_{3}\right), 22.8\left(\mathrm{CH}_{2}\right), 12.4\left(\mathrm{CH}_{3}\right)$;

B: ${ }^{1} \mathrm{H}$ NMR $\left(500 \mathrm{MHz}, \mathrm{CDCl}_{3}\right) \delta 7.33(\mathrm{~d}, J=8.2 \mathrm{~Hz}, 2 \mathrm{H}), 7.26(\mathrm{~d}, J=7.3 \mathrm{~Hz}, 1 \mathrm{H}), 7.21$ $-7.16(\mathrm{~m}, 1 \mathrm{H}), 6.96(\mathrm{td}, J=7.5,0.9 \mathrm{~Hz}, 1 \mathrm{H}), 6.89$ (dd, $J=15.2,10.4 \mathrm{~Hz}, 1 \mathrm{H}), 6.88(\mathrm{~d}, J=$ $8.2 \mathrm{~Hz}, 2 \mathrm{H}), 6.65(\mathrm{~d}, J=7.9 \mathrm{~Hz}, 1 \mathrm{H}), 5.97(\mathrm{dd}, J=10.2,4.1 \mathrm{~Hz}, 1 \mathrm{H}), 5.95(\mathrm{~d}, J=15.2$ $\mathrm{Hz}, 1 \mathrm{H}$ ), 5.75 (br d, $J=10.2,1 \mathrm{H}$ ), 4.08 (br dd, $J=6.7,4.3 \mathrm{~Hz}, 1 \mathrm{H}$ ), 3.76 (s, 3H), 3.66 (s, $3 \mathrm{H}), 3.61(\mathrm{~d}, J=7.3 \mathrm{~Hz}, 1 \mathrm{H}), 2.39(\mathrm{td}, J=10.4,2.1 \mathrm{~Hz}, 1 \mathrm{H}), 1.05(\mathrm{~s}, 3 \mathrm{H}), 1.02-0.95(\mathrm{~m}$, $1 \mathrm{H}), 0.83-0.73(\mathrm{~m}, 1 \mathrm{H}), 0.55$ (t, $J=7.3 \mathrm{~Hz}, 3 \mathrm{H}) ;{ }^{13} \mathrm{C} \mathrm{NMR}\left(75 \mathrm{MHz}, \mathrm{CDCl}_{3}\right) \delta 172.5(\mathrm{C})$, $166.6(\mathrm{C}), 149.4(\mathrm{CH}), 142.2(\mathrm{C}), 135.3(\mathrm{C}), 134.4(\mathrm{C}), 132.1(\mathrm{CH}), 131.2(\mathrm{CH}), 131.1$ $(\mathrm{CH}), 128.4(\mathrm{CH}), 127.1(\mathrm{CH}), 126.2(\mathrm{CH}), 125.2(\mathrm{CH}), 125.1(\mathrm{CH}), 123.9(\mathrm{CH}), 121.4$ (C), $59.1(\mathrm{CH}), 55.9(\mathrm{CH}), 52.0\left(\mathrm{CH}_{3}\right), 51.4\left(\mathrm{CH}_{3}\right), 42.9(\mathrm{CH}), 41.9(\mathrm{C}), 29.8\left(\mathrm{CH}_{3}\right), 22.8$ $\left(\mathrm{CH}_{2}\right), 12.4\left(\mathrm{CH}_{3}\right)$;

C: ${ }^{1} \mathrm{H}$ NMR $\left(500 \mathrm{MHz}, \mathrm{CDCl}_{3}\right) \delta 7.42(\mathrm{~d}, J=8.5 \mathrm{~Hz}, 2 \mathrm{H}), 7.27-7.20(\mathrm{~m}, 1 \mathrm{H}), 7.16-7.11$ (m, 1H), 7.09 (d, $J=8.5 \mathrm{~Hz}, 2 \mathrm{H}), 6.78(\mathrm{dd}, J=15.6,10.4 \mathrm{~Hz}, 1 \mathrm{H}), 6.82-6.77(\mathrm{~m}, 1 \mathrm{H})$, $6.13(\mathrm{br} \mathrm{d}, J=7.9 \mathrm{~Hz}, 1 \mathrm{H}), 6.01(\mathrm{dd}, J=10.5,4.5 \mathrm{~Hz}, 1 \mathrm{H}), 5.91(\mathrm{~d}, J=15.6 \mathrm{~Hz}, 1 \mathrm{H})$, $5.69(\mathrm{~d}, J=10.5 \mathrm{~Hz}, 1 \mathrm{H}), 3.98(\mathrm{dd}, J=9.9,4.5 \mathrm{~Hz}, 1 \mathrm{H}), 3.76(\mathrm{~s}, 3 \mathrm{H}), 3.71(\mathrm{~s}, 3 \mathrm{H}), 3.49$ $(\mathrm{d}, J=9.9 \mathrm{~Hz}, 1 \mathrm{H}), 2.32(\mathrm{td}, J=11.0,2.7 \mathrm{~Hz}, 1 \mathrm{H}), 1.36-1.24(\mathrm{~m}, 2 \mathrm{H}), 1.27(\mathrm{~s}, 3 \mathrm{H}), 0.74$ (t, $J=7.3 \mathrm{~Hz}, 3 \mathrm{H})$; 
D: ${ }^{1} \mathrm{H}$ NMR $\left(500 \mathrm{MHz}, \mathrm{CDCl}_{3}\right) \delta$ 7.38-7.34 (m, 3H), 7.24-7.18 (m, 3H), $7.04(\mathrm{~d}, J=8.2$ $\mathrm{Hz}, 1 \mathrm{H}), 6.76(\mathrm{dd}, J=15.6,10.4 \mathrm{~Hz}, 1 \mathrm{H}), 5.83$ (d, $J=15.6 \mathrm{~Hz}, 2 \mathrm{H}), 5.79$ (br dd, $J=$ 10.5, 4.0 Hz, 1H), 5.59 (br d, $J=10.5 \mathrm{~Hz}, 1 \mathrm{H}), 4.25-4.21(\mathrm{~m}, 1 \mathrm{H}), 3.94$ (d, $J=7.0 \mathrm{~Hz}$, 1H), 3.76 (s, 3H), 3.69 (s, 3H), 2.17 (td, $J=10.4,3.0 \mathrm{~Hz}, 1 \mathrm{H}), 1.17$ (s, 3H), 0.68-0.54 (m, $2 \mathrm{H}), 0.54(\mathrm{t}, J=7.3 \mathrm{~Hz}, 3 \mathrm{H}) ;{ }^{13} \mathrm{C} \mathrm{NMR}\left(75 \mathrm{MHz} \mathrm{CDCl}_{3}\right) \delta 173.6,166.6,149.6,142.3$, $139.3,134.9,132.8,131.3,131.2,127.8,127.1,126.4,126.0,125.0,123.8,121.6,58.3$, $56.1,52.1,51.5,42.0,41.4,30.3,22.7,12.6$

LRMS (ESI) $m / z$ (relative intensity): 377.1 (14), $519.2\left([\mathrm{M}+\mathrm{Na}]^{+}, 100\right)$; HRMS (ESI) $\mathrm{m} / z$ Calcd for $\left[\mathrm{C}_{27} \mathrm{H}_{29} \mathrm{BrNaO}_{4}\right]^{+}\left([\mathrm{M}+\mathrm{Na}]^{+}\right): 519.1141$. Found: 519.1153; Anal. Calcd for $\mathrm{C}_{27} \mathrm{H}_{29} \mathrm{BrO}_{4}$ (mixture of diastereomers): C, 65.19; H, 5.88. Found: C, 65.10; H, 5.96. 